\title{
Effect of the hydration on the biomechanical properties in a fibrin-agarose tissue-like model
}

\author{
Giuseppe Scionti, ${ }^{1}$ Monica Moral, ${ }^{2}$ Manuel Toledano, ${ }^{3}$ Raquel Osorio, ${ }^{3}$ Juan D. G. Durán, ${ }^{2}$ Miguel \\ Alaminos, ${ }^{1}$ Antonio Campos, ${ }^{1}$ Modesto T. López-López ${ }^{2}$ \\ ${ }^{1}$ Department of Histology, University of Granada, Avenida de Madrid 11, 18012, Granada, Spain \\ ${ }^{2}$ Department of Applied Physics, University of Granada, Avenida Fuente Nueva, 18071, Granada, Spain \\ ${ }^{3}$ Department of Stomatology, University of Granada, Colegio Máximo, 18071, Granada, Spain
}

Received 27 June 2013; revised 8 August 2013; accepted 12 August 2013

Published online 00 Month 2013 in Wiley Online Library (wileyonlinelibrary.com). DOI: 10.1002/jbm.a.34929

Abstract: The effect of hydration on the biomechanical properties of fibrin and fibrin-agarose (FA) tissue-like hydrogels is reported. Native hydrogels with approximately $99.5 \%$ of water content and hydrogels with water content reduced until $90 \%$ and $80 \%$ by means of plastic compression (nanostructuration) were generated. The biomechanical properties of the hydrogels were investigated by tensile, compressive, and shear tests. Experimental results indicate that nanostructuration enhances the biomechanical properties of the hydrogels. This improvement is due to the partial draining of the water that fills the porous network of fibers that the plastic compression generates, which produces a denser material, as confirmed by scanning electron microscopy. Results also indicate that the characteristic compressive and shear param- eters increase with agarose concentration, very likely due to the high water holding capacity of agarose, which reduces the compressibility and gives consistency to the hydrogels. However, results of tensile tests indicate a weakening of the hydrogels as agarose concentration increases, which evidences the anisotropic nature of these biomaterials. Interestingly, we found that by adjusting the water and agarose contents it is possible to tune the biomechanical properties of FA hydrogels for a broad range, within which the properties of many native tissues fall. (c) 2013 Wiley Periodicals, Inc. J Biomed Mater Res Part A: 00A:000-000, 2013.

Key Words: fibrin, agarose, hydrogel, mechanical properties, scaffold

How to cite this article: Scionti G, Moral M, Toledano M, Osorio R, Durán JDG, Alaminos M, Campos A, López-López MT. 2013. Effect of the hydration on the biomechanical properties in a fibrin-agarose tissue-like model. J Biomed Mater Res Part A 2013: 00A: 000-000.

\section{INTRODUCTION}

Hydrogels are networks of polymer chains in which water is the dispersion medium that can be designed to meet defined swelling and mechanical properties. In addition, many hydrogels are biocompatible, which makes possible their potential use in a variety of biomedical applications. ${ }^{1}$ Furthermore, some hydrogels have a fibrillose microstructure, which may be similar to the extracellular matrix of natural tissues. Therefore, hydrogels can be used as scaffolds for tissue engineering applications. ${ }^{1}$ Unfortunately, most hydrogels are much weaker than native tissues from a mechanical viewpoint, which limits their practical use in tissue engineering. ${ }^{2}$ The poor mechanical properties of these hydrogels are caused by several reasons, including the random alignment of fibers and the high water content of the materials. ${ }^{3}$

Hydrogels used for tissue engineering applications can be classified into two groups: hydrogels made from natural polymers and hydrogels made from synthetic polymers. ${ }^{4}$ One of the most commonly used natural polymers is fibrin, which is a biodegradable protein involved in the natural process of tissue repair upon injury. Fibrin forms long fibril chains which are very flexible, and possess high mechanical strength. ${ }^{5}$ Furthermore, fibrin could form quite big threedimensional structures by connection of different fibrin fibrils. Such organization makes fibrin structures among the most resilient within the world of natural protein. ${ }^{6}$ These characteristics of fibrin give fibrin hydrogels a potential for several tissue engineering applications. ${ }^{7}$ However, new methods and techniques should be developed to improve the mechanical properties of fibrin hydrogels. In this regard, our research group described the combination of fibrin with agarose as a straightforward method to improve the mechanical properties of fibrin hydrogels in tissue engineering. ${ }^{8,9}$ Agarose is a polysaccharide extracted from marine

Correspondence to: M. T. López-López; e-mail: modesto@ugr.es

Contract grant sponsor: Spanish Plan Nacional de Investigación Científica, Desarrollo e Innovación Tecnológica (I+D+i) from the Spanish Minis try of Economy and Competitiveness (Instituto de Salud Carlos III); contract grant number: FIS PI11/1582 and FIS PI11/2668

Contract grant sponsor: Junta de Andalucía (Consejería de Innovación, Ciencia y Empleo, Proyectos de Excelencia); contract grant number: P10CTS-6060 and P09-FQM-4787 
red algae that forms thermally reversible biocompatible gels. ${ }^{10}$ More recently, a nanostructuration method based on the application of a plastic compression resulted in the modulation of the mechanical properties of fibrin-agarose (FA) gels. ${ }^{9}$

FA hydrogels have been successfully used for the preparation of different bioengineered tissues, including cornea, skin, oral mucosa, and peripheral nerve. ${ }^{8,11-14}$ Dehydration by plastic compression proved to be an efficient way to improve the mechanical properties of FA hydrogels at $0.1 \%$ of agarose concentration. ${ }^{9}$ Nevertheless, there is not any reported study that thoroughly analyzes the main variables of the preparation protocol of this kind of hydrogels, that is, the agarose concentration and the water content.

The aim of this work is to analyze in details the effect of hydration grade and agarose content on the biomechanical properties of FA hydrogels. For this aim, we prepared several FA hydrogels with different agarose content and aliquots of each of them were subjected to plastic compression of different strength in order to vary the hydration grade. The mechanical behavior of the obtained hydrogels was measured under tensile, compressive and shear loads, to achieve a complete mechanical characterization of the biomaterials and a deeper knowledge of their potential for tissue engineering applications.

\section{METHODOLOGY}

\section{Preparation of hydrogels}

For the production of fibrin and FA hydrogels we used a mixture of human fibrin and agarose. For this aim, we adapted the method reported in Ref. 8. Briefly, we produced a fibrin gel by mixing $3.8 \mathrm{~mL}$ of human plasma of blood donors (kindly provided by Dr. Fernandez-Montoya, Human Tissue Bank of Granada) and $75 \mu \mathrm{L}$ of tranexamic acid in a petri dish. Note that tranexamic acid is an anti-fibrinolytic agent that prevents degradation of the scaffold. Then, 0.25 $\mathrm{mL}$ of a solution of $2 \% \mathrm{CaCl}_{2}$ was added to the solution to activate the fibrin polymerization process. Finally, a given amount of type VII agarose in PBS $(0.02 \mathrm{~g} / \mathrm{mL}$ of concentration) was added to the mixture, to a final agarose concentration of $0,0.1,0.2,0.3,0.4$, and $0.5 \%$, and the final volume was adjusted to $5 \mathrm{~mL}$ with Dulbecco's Modified Eagle's Medium. Gels were then kept at $37^{\circ} \mathrm{C}$ until complete gelation. As control, a gel consisting only of type VII agarose in PBS $(0.02 \mathrm{~g} / \mathrm{mL}$ of agarose) was used. We will refer to this gel as pure agarose gel. Once generated, some fibrin and FA hydrogels were subjected to plastic compression (nanostructuration) of different strength to partially dehydrate them and to obtain fibrin and FA gels with different levels of hydration (approximately 99.5, 90, and 80\%). To be precise, we placed the samples between a couple of filter nylon membranes with a $0.22 \mu \mathrm{m}$ pore size, and compressed them between a pair of sterile Whatman $3 \mathrm{~mm}$ absorbent papers under a flat glass surface. We applied uniform mechanical pressure to the hydrogels until they reached the weight corresponding to the desired water content percentage (80 or $90 \%$ ). By applying these methods, we obtained: (1) fully hydrated hydrogels with a water con- tent of approximately 99.5\%, (2) nanostructured hydrogels with a water concentration of $90 \%$, and (3) nanostructured hydrogels with $80 \%$ of water content.

The combination of the two parameters (agarose content and hydration grade) gave rise to the production of 18 different formulations of fibrin and FA hydrogels. We produced 11 samples for each of the 18 formulations, and tested four of them under tensile stress, four under compressive load, and three under shear stress. Results shown in this article correspond to the average of the measurements. Note that all the prepared hydrogels had a disk-like shape and that the direction of nanostructuration coincided with the main axis of the disk-like sample (Fig. 1).

\section{Microscopic analysis of fibrin and FA hydrogels}

We characterized the structure of the hydrogels from a microscopic viewpoint by means of scanning electron microscopy (SEM). For this aim, we sectioned the hydrogels in longitudinal and transverse planes.

We measured the thickness of the samples with the help of a light microscope (Nikon Instruments). For this, we placed each sample on a microscopy slide, focused on the top of both the sample and the slide, and determined the difference in the $Z$-axis focus between the two positions.

\section{Rheological tests under shear stress}

We evaluated the rheological properties of the hydrogels under shear stress with a Haake Mars III rheometer. The measuring geometry consisted of a pair of parallel plates, with serrated surfaces in order to avoid wall slip. In each case, we adjusted the gap between the plates to the thickness of the respective sample. We conducted all the shear measurements at $37.0 \pm 0.1^{\circ} \mathrm{C}$ under a water vaporsaturated atmosphere. We analyzed two regimes of simple shear: steady-state regime and dynamic or oscillatory regime. In steady-state measurements, we subjected the samples to a ramp of increasing shear strain, $\gamma$, and the corresponding shear stress, $\sigma$, was monitored. In these experiments, the shear strain was increased at a constant rate of $0.01 \mathrm{~s}^{-1}$

From $\sigma$ versus $\gamma$ curves, we obtained the shear (or rigidity) modulus of the hydrogels, which is defined as $G=\sigma / \gamma$. In this work, we determined the shear modulus as the slope of the initial linear part of the $\sigma$ versus $\gamma$ curves. In dynamic (oscillatory) measurements we subjected the samples to a sinusoidal shear stress given by $\sigma=\sigma_{0} \sin (2 \pi f t)$, and the corresponding (oscillatory) shear strain, $\gamma=\gamma_{0} \sin (2 \pi f t+\delta)$ was monitored. In these equations, $f(\mathrm{~Hz})$ is the frequency, $\sigma_{0}$ and $\gamma_{0}$ are the stress and strain amplitudes, respectively, and $\delta$ is a lag angle between the phases of the stress and the strain. From such a kind of experiments, we obtained the so-called viscoelastic moduli, which completely characterize the viscoelasticity of the materials under shear: the elastic or storage modulus $\left(G^{\prime}\right)$ and the viscous or loss modulus $\left(G^{\prime \prime}\right)$.

We performed two different kinds of oscillatory tests: amplitude sweeps and frequency sweeps. In amplitude sweeps, the frequency was fixed at $f=1 \mathrm{~Hz}$ and the 


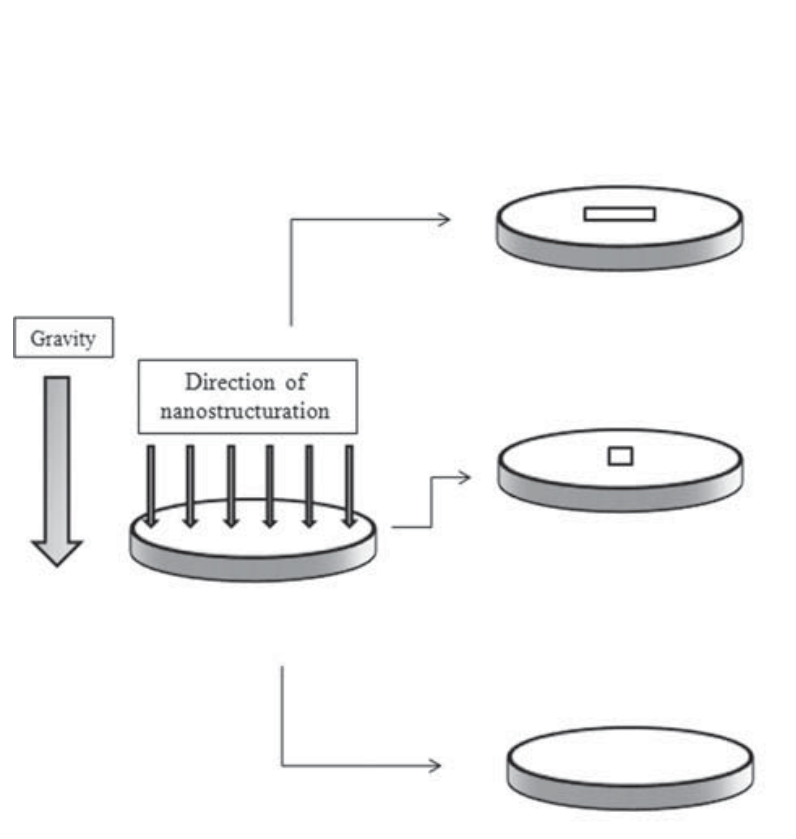

c)

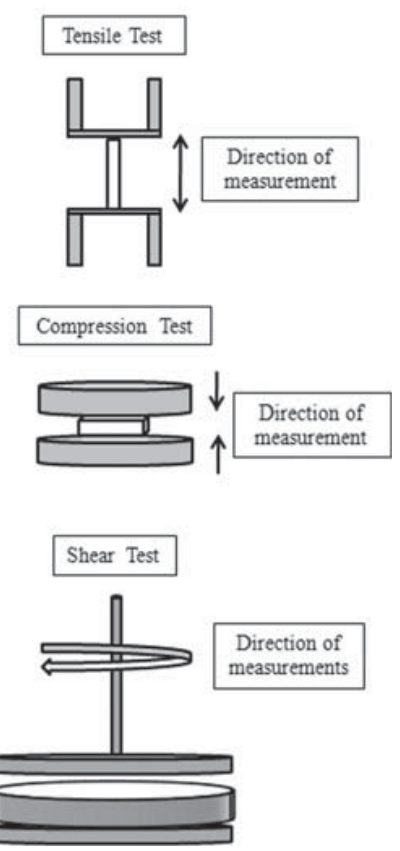

FIGURE 1. Schematic representation of the biomechanical analysis made in this work. (a) Sketch of the direction of nanostructuration with respect to the sample shape. (b) Specimens were cut for tensile and compression tests, whereas the full disk-like sample was used in shear tests. (c) Sample orientation with respect to the direction of measurement in the different tests.

amplitude of the applied stress, $\sigma_{0}$, was increased until a value high enough to reach the non-linear viscoelastic region (NLVR). The NLVR is characterized by a non-linear response of the viscoelastic moduli $\left(G^{\prime}\right.$ and $\left.G^{\prime \prime}\right)$, for $\sigma_{0}$ values larger than a critical shear stress amplitude $\left(\sigma_{\mathrm{c}}\right)$. For $\sigma_{0}$ values smaller than $\sigma_{\mathrm{c}}$, the viscoelastic moduli are almost independent of $\sigma_{0}$ (a pseudoplateau is usually observed when $G^{\prime}$ and $\mathrm{G}^{\prime \prime}$ are plotted as a function of $\sigma_{0}$ ). This region is commonly known as viscoelastic linear region (VLR). Once we determined the values of $\sigma_{0}$ pertaining to the VLR, we carried out frequency sweeps. In these measurements, $\sigma_{0}$ was maintained at a constant value well into the VLR, and a frequency sweep was carried out. As a result, we obtained the frequency dependence of $G^{\prime}$ and $G^{\prime \prime}$. We limited the frequency range to a maximum of $7 \mathrm{~Hz}$ in order to make negligible the influence of sample inertia in the performed measurements. ${ }^{15}$

\section{Tensile and compression tests}

For the tensile tests we used an electromechanical material testing machine (Instron, Model3345-K3327). For these tests, we cut the disk-like hydrogels into cuboid samples of a width of $10 \mathrm{~mm}$, a length of $60 \mathrm{~mm}$ and a height equal to the thickness of the disk-like hydrogels (Fig. 1). The samples were oriented with their length along the direction of tension (Fig. 1). We fixed the distance between the clamps of the testing machine to $15 \mathrm{~mm}$. Therefore, the volume of the samples that underwent tensile stress had dimensions of 10 $\mathrm{mm} \times 15 \mathrm{~mm} \times$ thickness of the sample. The remaining parts of the samples were gripped between the clamps. The tests were run at a constant strain rate of $5 \mathrm{~mm} / \mathrm{min}$ at room temperature. The Young's modulus was calculated as the tangent modulus of the initial, linear portion of the stress-strain curve of each experimental run, while the stress at break $\left(\sigma_{\text {break }}\right)$ and the strain at break $\left(\varepsilon_{\text {break }}\right)$ values were determined by selecting the point of the stressstrain curve where the fracture occurred.

We used the same electromechanical material testing machine (Instron, Model3345-K3327) for the uniaxial compression tests. In this case, the hydrogels were cut into cuboid samples of a width of $15 \mathrm{~mm}$, a length of $15 \mathrm{~mm}$ and a height equal to the thickness of the disk-like hydrogels (Fig. 1). The samples were placed between two impermeable, unlubricated platens, oriented with their thickness along the direction of compression (Fig. 1), and submitted to unconfined compression at a constant strain rate of 1 $\mathrm{mm} / \mathrm{min}$ at room temperature. The elastic compressive modulus was determined from the slope of the stress-strain curve at $15 \%$ of strain.

\section{RESULTS}

\section{Microscopic analysis of fibrin and FA hydrogels}

From SEM observations it was clear that the effect of nanostructuration was a qualitative decrease of porosity and, thus, an increase of fiber density in the hydrogels (Fig. 2). In addition, in the non-nanostructured hydrogels there was an approximately random alignment of the fibers. On the contrary, for hydrogels subjected to nanostructuration of 
(a)

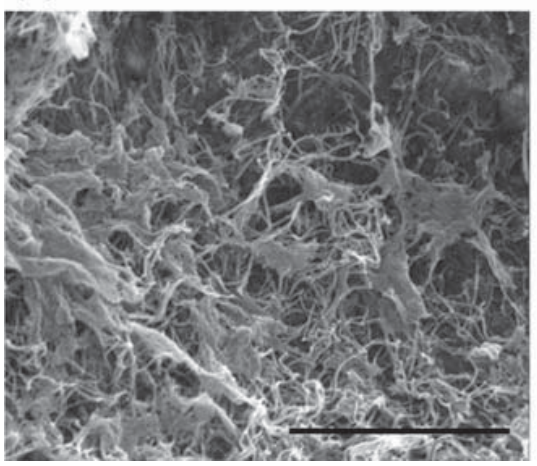

(b)

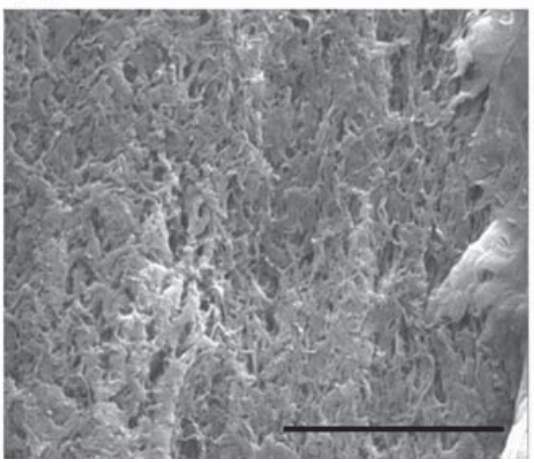

(c)

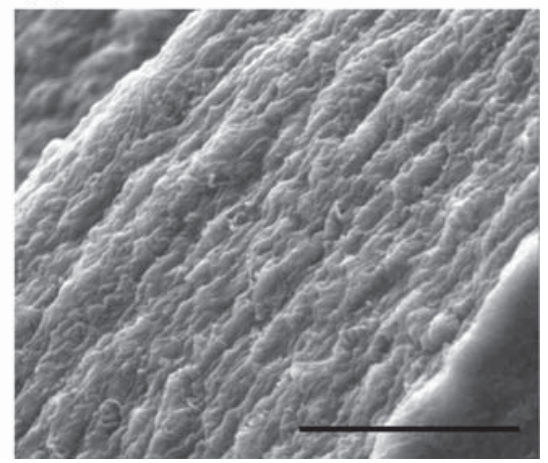

FIGURE 2. Illustrative analysis of FA hydrogels with $0.3 \%$ of agarose (FA $0.3 \%$ hydrogels) by SEM (transversal section). (a) Non-nanostructured hydrogel, with $99.48 \%$ of water content; (b) hydrogel nanostructured down to $90 \%$ of hydration; (c) hydrogel nanostructured down to $80 \%$ of hydration. Scale bar: $20 \mu \mathrm{m}$.

increasing intensity, a progressive distribution of fibrin fibers in parallel layers was seen, especially in hydrogels with $80 \%$ of water content (Fig. 2). Concerning the agarose content, and in comparison with the homogeneous network of fibrin fibers observed in fibrin gels, this network was disrupted by the presence of agarose spots throughout the F3 hydrogel (Fig. 3).

Regarding the thickness of the hydrogels, we obtained that this parameter decreased dramatically for the nanostructured samples with respect to the non-nanostructured

T1 (native) ones (Table I). We also obtained that the thickness was smaller when the nanostructuration was stronger. With respect to the effect of agarose concentration on the thickness, our results indicate that the latter increased moderately with the former for both non-nanostructured and nanostructured hydrogels (Table I).

\section{Rheological tests under shear stress}

Steady-state measurements. We observed two different trends for the dependences of the shear modulus, $G$, on the

F4 hydration grade and the agarose content (Fig. 4). On the one hand, $G$ of the hydrogels increased with the agarose concentration. On the other hand, $G$ of the hydrogels decreased when the hydration grade increased. Thus, the highest value of the shear modulus was obtained for the FA $0.5 \%$ hydrogels nanostructured down to $80 \%$ of hydration.

Oscillatory measurements. The curves of $G^{\prime}$ and $G^{\prime \prime}$ versus $\sigma_{0}$ at a frequency of $1 \mathrm{~Hz}$ showed an initial pseudoplateau, followed by a sharp drop, as illustrated in Figure 5 for the non-nanostructured FA $0.5 \%$ hydrogels-the rest of the fibrin and FA hydrogels show a similar trend (curves not shown here for brevity). The initial pseudoplateau was associated to the VLR, as mentioned above. As observed, in this region, $G^{\prime}$ is almost one order of magnitude higher than $G^{\prime \prime}$, something that holds for any of the hydrogels studied in our work.

From curves like these shown in Figure 8, we obtained the average value of the viscoelastic moduli $\left(G^{\prime}\right.$ and $\left.G^{\prime \prime}\right)$ pertaining to the VLR of the different fibrin and FA hydrogels (Fig. 6). As observed, the values of both $G^{\prime}$ and $G^{\prime \prime}$ increased with the agarose content in the hydrogels, for all the hydration grades. With respect to the effect of hydration, we observed a trend of the viscoelastic moduli to increase (a)

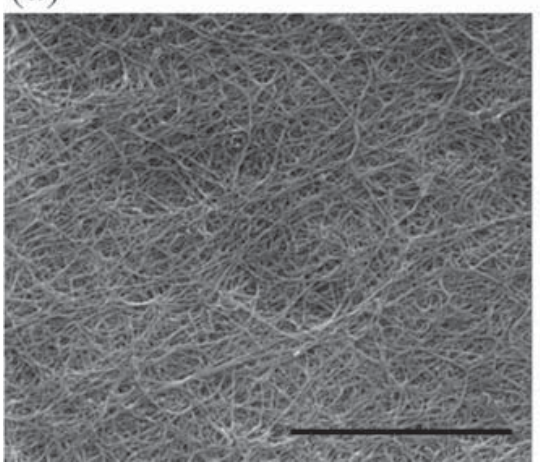

(b)

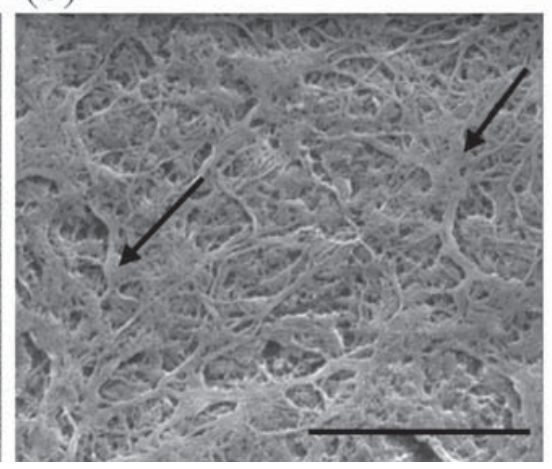

(c)

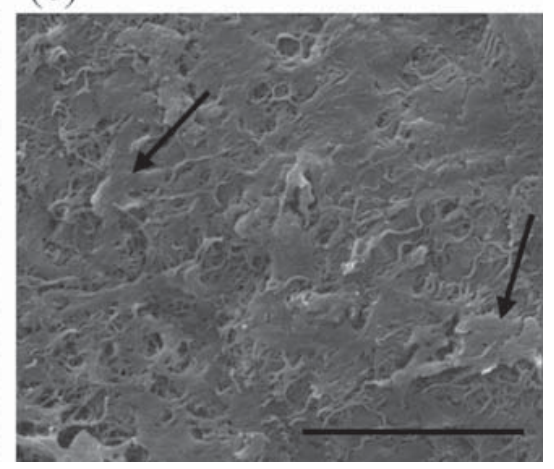

FIGURE 3. Illustrative SEM images of fibrin and FA hydrogels nanostructured down to $90 \%$ of hydration grade. Agarose concentration: (a) $0 \%$; (b) $0.3 \%$, (c) $0.5 \%$. Some agarose spots are highlighted with arrows. Scale bar: $20 \mu \mathrm{m}$. 
TABLE I. Thickness $(\mu \mathrm{m})$ of the Fibrin and FA Hydrogels

\begin{tabular}{lccccc}
\hline & Fibrin & FA 0.1\% & FA 0.2\% & FA 0.3\% & FA 0.4\% \\
\hline Non-nanostructured hydrogel & 2000 & 3000 & 3500 & 3700 & 3700 \\
Hydrogel nanostructured to 90\% of hydration & 150 & 150 & 175 & 230 & 230 \\
Hydrogel nanostructured to 80\% of hydration & 60 & 60 & 100 & 100 & 110 \\
\hline
\end{tabular}

when the water content in the hydrogels decreased. Note that these trends were similar to these shown by the shear modulus.

Concerning the dependence of the viscoelasticity of the hydrogels corresponding to the VLR with the frequency of the oscillatory shear stress, all the fibrin and FA hydrogels showed a similar trend, characterized by a slight increase of

F7 both $G^{\prime}$ and $G^{\prime \prime}$ with frequency, as depicted in Figure 7 for the non-nanostructured FA $0.5 \%$ hydrogel. Finally, the analysis of control pure agarose gel revealed that the values of $G^{\prime}$ and $G^{\prime \prime}$ of this gel were equal to 8.80 and $0.27 \mathrm{kPa}$ respectively.

\section{Tensile and compression tests}

Tensile Young's modulus. Our results showed an important influence of water and agarose contents on the tensile Young's moduli of the fibrin and FA hydrogels (Fig. 8). For all agarose contents, the hydrogels at $80 \%$ of hydration showed the highest values of tensile Young's modulus, while the non-nanostructured samples showed the smallest values of this parameter. In particular, the values of the Young's modulus of samples with $80 \%$ of hydration were more than two times higher than the values of samples with $90 \%$ of hydration, and up to 40 times higher than the values shown by the non-nanostructured hydrogels. Regarding the effect of agarose content, for all hydration grades the Young's modulus decreased when agarose content increased. Consequently, the highest value of the Young's modulus $(0.46 \pm 0.05 \mathrm{MPa})$ was associated to fibrin $(0 \%$ of agarose $)$

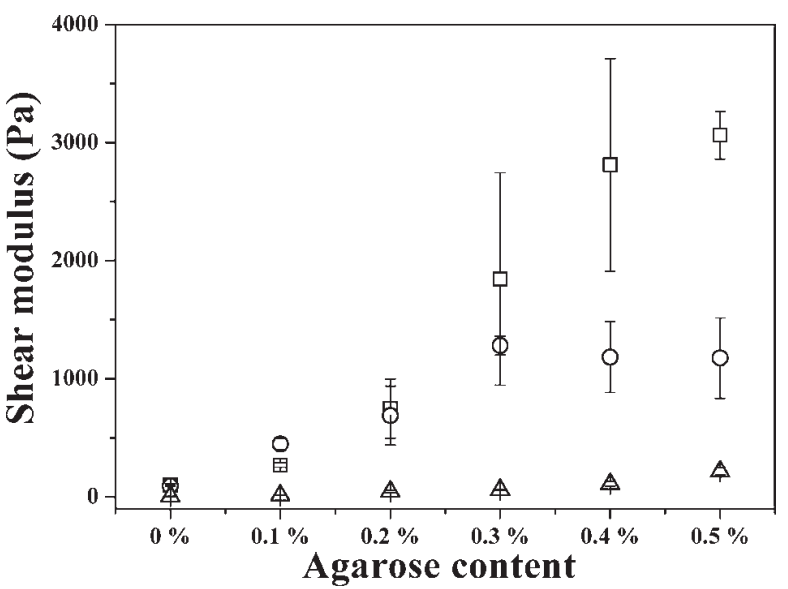

FIGURE 4. Shear modulus ( $G$ ) of fibrin and FA hydrogels plotted as a function of agarose concentration. $\Delta$ : Non-nanostructured hydrogels; $\bigcirc$ : Nanostructured hydrogels with $90 \%$ of hydration; $\square$ : Nanostructured hydrogels with $80 \%$ of hydration. Error bars correspond to standard deviations. hydrogel with $80 \%$ of hydration. The analysis of control pure agarose gel revealed that the value of the Young's Modulus of this gel was equal to $0.03 \mathrm{MPa}$.

Tensile stress and strain at break. The analysis of the results of the stress at break showed that the fracture of the samples with $80 \%$ of hydration occurred at higher values of tensile stress, as compared to samples with higher water content, independently of the agarose content (Fig. 9). In particular, the values of the samples with $90 \%$ of hydration were less than half of the values of the samples with $80 \%$ of hydration at the same agarose concentration. Concerning the non-nanostructured samples, these tended to fracture at very low stresses in comparison with samples with lower percentages of water. With respect to the effect of agarose content, the stress at break showed a clear tendency to decrease as the agarose concentration increased. Therefore, similarly to the tensile Young's modulus, the highest value of the stress at break was obtained for the fibrin $(0 \%$ of agarose) hydrogel with $80 \%$ of hydration $(0.61 \pm 0.13 \mathrm{MPa})$. The analysis of control pure agarose gel revealed that the value of the stress at break of these gels was equal to $0.007 \mathrm{MPa}$. For the strain at fracture, the same trends were found (not shown here for brevity).

Compressive elastic modulus. Similarly to tensile tests, compression tests showed an important influence of hydration grade and agarose concentration on the mechanical

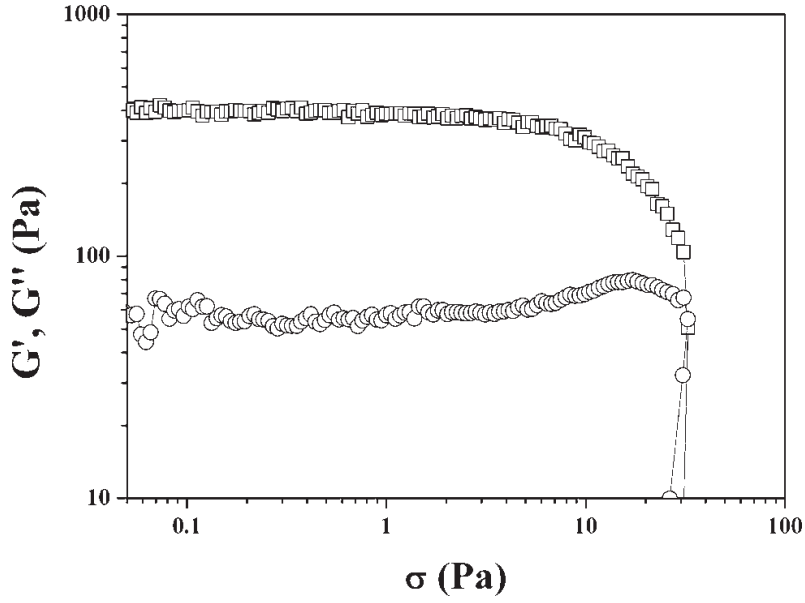

FIGURE 5. Illustrative representation of the viscoelastic moduli ( $G$ and $G^{\prime \prime}$ ) as a function of the stress amplitude for the nonnanostructured FA $0.5 \%$ hydrogel. Measurements were performed by subjecting the samples to sinusoidal shear stresses at a frequency of $1 \mathrm{~Hz}$. $\square$ : elastic modulus $\left(G^{\prime}\right)$; $\bigcirc$ : viscous modulus $\left(G^{\prime \prime}\right)$. 

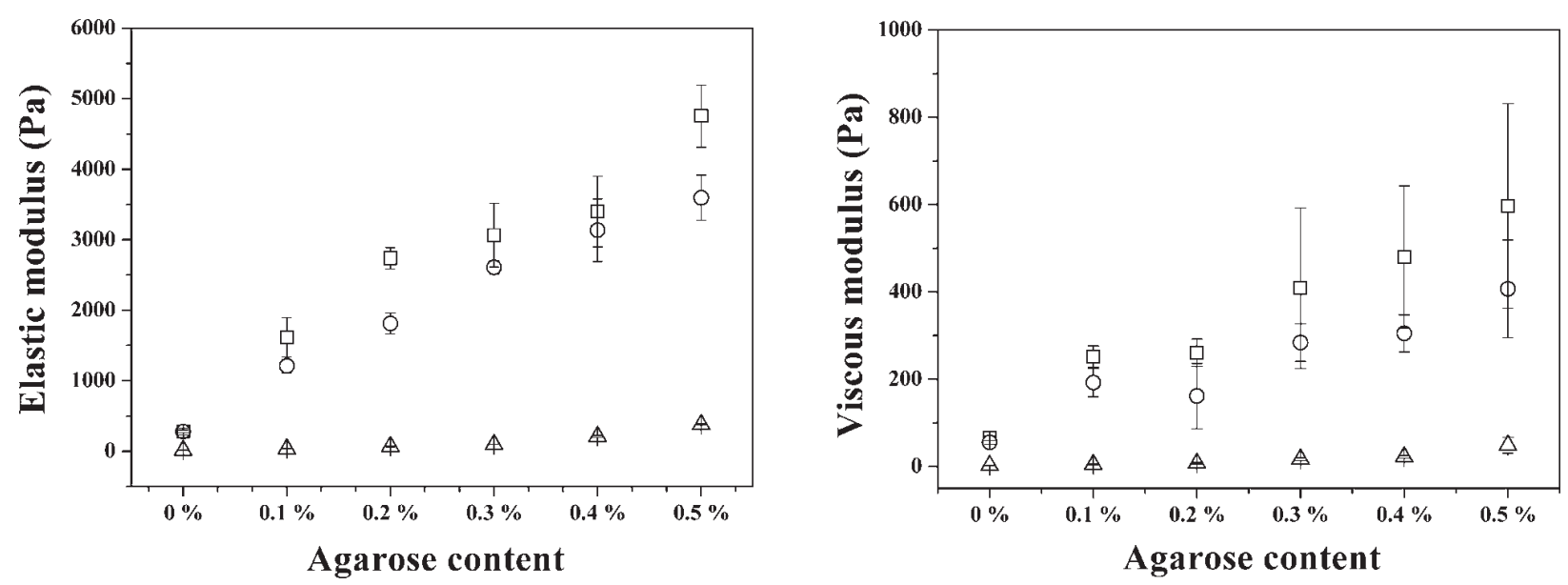

FIGURE 6. Average values of the viscoelastic moduli within the VLR of fibrin and FA hydrogels, plotted as a function of agarose concentration. $\Delta$ : Non-nanostructured hydrogels; $\bigcirc$ : Nanostructured hydrogels with $90 \%$ of hydration; $\square$ : Nanostructured hydrogels with $80 \%$ of hydration. (a)elastic modulus $\left(G^{\prime}\right)$; (b) viscous modulus $\left(G^{\prime \prime}\right)$. Error bars correspond to standard deviations.

behavior of the fibrin and FA hydrogels. Results demonstrate that the hydrogels at $80 \%$ of hydration had the highest values of elastic modulus, for all agarose concentrations

F10 (Fig. 10). Regarding the effect of agarose content, the compressive elastic modulus increased when the concentration of agarose increased, contrarily to the case of the tensile Young's modulus. The analysis of control pure agarose gel revealed that the value of the compressive elastic modulus of this gel was equal to $0.08 \mathrm{MPa}$.

\section{DISCUSSION}

The addition of a quantity of agarose to fibrin hydrogels and the application of plastic compression (nanostructuration) to dehydrate them, have previously shown to be effective methods in modulating the mechanical and optical properties of fibrin-based biomaterials. ${ }^{8,9}$ However, to our knowledge, a complete biomechanical analysis of FA tissue-

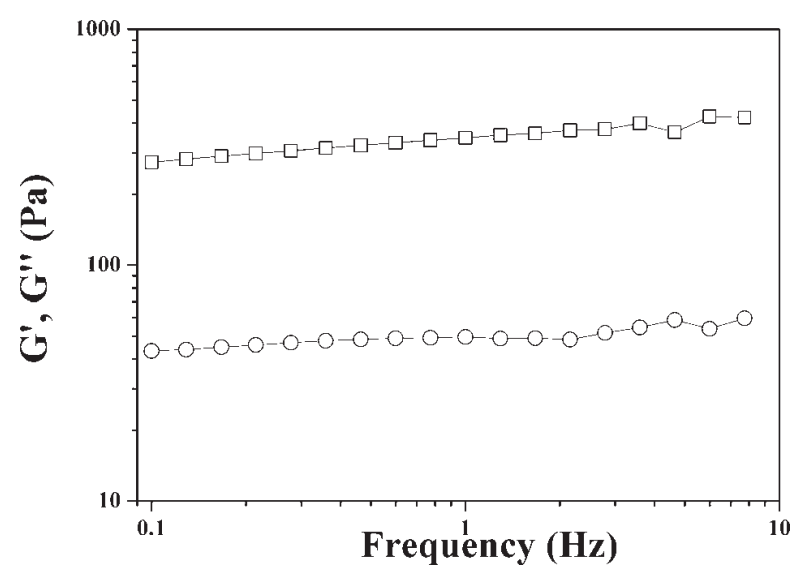

FIGURE 7. Illustrative representation of the viscoelastic moduli as a function of frequency for the non-nanostructured FA $0.5 \%$ hydrogel. Measurements were performed by subjecting the samples to sinusoidal shear stresses of fixed amplitude within the VLR. $\square$ : elastic modulus $\left(G^{\prime}\right)$; $\bigcirc$ : viscous modulus $\left(G^{\prime \prime}\right)$ like model has not been conducted to date. In this work, we have studied the effect of agarose content and hydration grade on the biomechanical behavior of fibrin-based hydrogels under tensile, compressive and shear stresses, in order to determine the most appropriate biomaterials for the generation of artificial tissues by tissue engineering. Our results showed that both agarose content and hydration grade have an important effect on the biomechanical properties of the hydrogels, and both parameters should therefore be controlled during the ex vivo fabrication process of human tissues.

\section{Effect of nanostructuration}

As the hydration grade of the biomaterial decreases, there is a progressive and significant increase in the elastic moduli

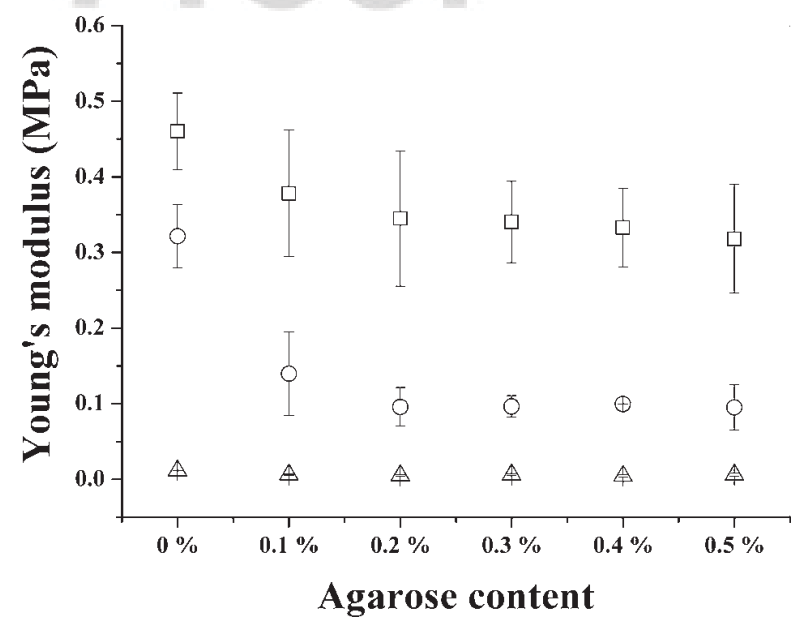

FIGURE 8. Tensile Young's modulus of fibrin and FA hydrogels plotted as a function of agarose content. $\Delta$ : Non-nanostructured hydrogels; $\bigcirc$ : Nanostructured hydrogels with $90 \%$ of hydration; $\square$ : Nanostructured hydrogels with $80 \%$ of hydration. Error bars correspond to standard deviations. 


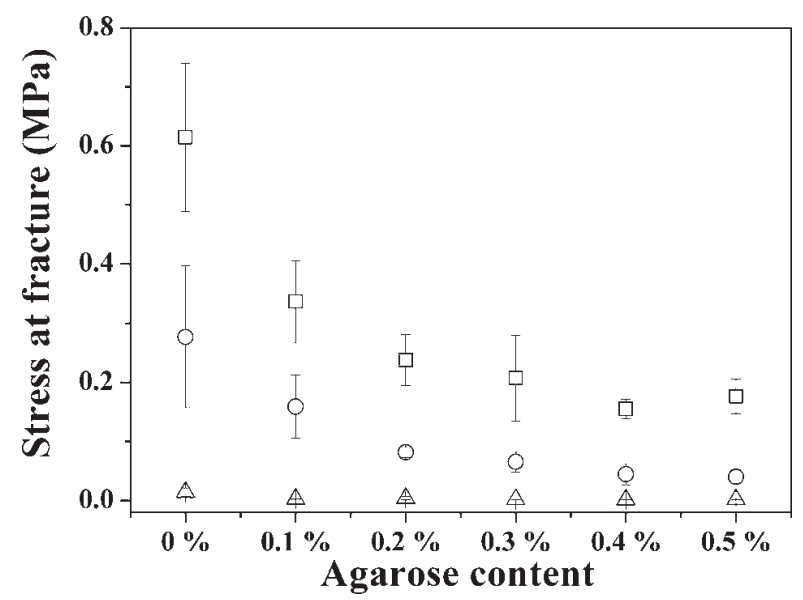

FIGURE 9. Tensile stress at fracture of fibrin and FA hydrogels plotted as a function of agarose content. $\Delta$ : Non-nanostructured hydrogels; $\bigcirc$ : Nanostructured hydrogels with $90 \%$ of hydration; $\square$ : Nanostructured hydrogels with $80 \%$ of hydration. Error bars correspond to standard deviations.

under tensile and compressive stresses, and a similar increase of the viscoelastic moduli under shear stresses. In addition, the application of plastic compression also increases the tensile stress and strain at failure. This can be interpreted in terms of the modifications in the threedimensional structure that the nanostructuration provokes on fibrin fibers and probably on agarose as well: the plastic compression drains part of the water that fills the spaces inside the porous network of fibers, producing a denser and stiffer material, as seen in Figure 2; the closer distance between fibers may enhance the formation of covalent interactions and hydrogen bonding between the molecules of the biomaterial, which additionally contributes to the increase of the characteristic biomechanical parameters of the hydrogels, as previously demonstrated for fibrin hydrogels. ${ }^{16}$

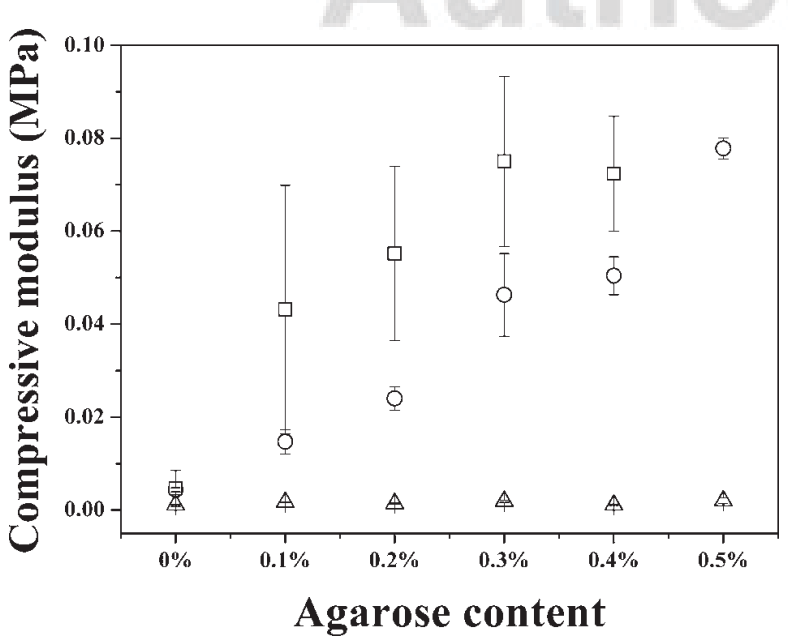

FIGURE 10. Elastic compressive modulus at $15 \%$ of strain of fibrin and FA hydrogels, plotted as a function of agarose concentration. $\Delta$ Non-nanostructured hydrogels; $\bigcirc$ : Nanostructured hydrogels with $90 \%$ of hydration; $\square$ : Nanostructured hydrogels with $80 \%$ of hydration. Error bars correspond to standard deviations.

\section{Effect of agarose content}

Properties under shear stress and compression stress. The addition of a defined quantity of agarose to the fibrin hydrogels produced more consistent and easier-tohandle biomaterials, which showed to possess higher elastic moduli under shear and compressive stresses. In these tests, the characteristic biomechanical parameters increase almost linearly until $0.3 \%$ of agarose concentration, the increase being weaker for higher agarose content. This is likely because agarose has great water holding capacity, which apparently makes the biomaterial more resistant to compressive and shear stress. In fact, hydrogels made with pure type VII agarose at 2\%, tested under compressive and shear stresses, showed values of compressive elastic modulus, storage modulus and loss modulus higher than any of the fibrin and FA hydrogels of the present study. The high water holding capacity of agarose is also evidenced by the increase of the thickness of the samples with agarose content (Table I). These results are in agreement with previous reports by our research group suggesting that the viscoelastic moduli tend to increase with increasing agarose concentrations. $^{9}$

Properties under tensile stress. In contrast, tensile tests showed that the addition of agarose provokes a decrease of the elastic modulus, the stress at fracture and the strain at fracture under tensile stress, until an asymptotic value that is reached approximately at $0.3 \%$ of agarose, depending on the hydration grade. Interestingly, the effect of agarose content in tensile tests differs from the results obtained in compressive and shearing tests. This finding suggests that the mechanical properties of fibrin and FA hydrogels are strongly dependent on the direction of the forces applied to these biomaterials, implying that these gels could be anisotropic upon nanostructuration. In this regard, previous works demonstrated that fibrin fibers have the capability of distributing the forces throughout the fibril network under stress, so that the strain concentrations may be shared by all fibrils, resulting in a sequential stiffening of the fibrin hydrogels. ${ }^{17,18}$ It is, therefore, evident that the direction of the fibers within the biomaterial will influence the behavior of the mesh upon mechanical testing. For this reason, the different orientation of the samples in the different measurements with respect to the direction of gravity and nanostructuration forces during preparation could explain these results. To be precise, in compression tests the stress is applied in the same direction as the gravity and nanostructuration forces, whereas in tensile tests the force is applied perpendicularly to these forces (Fig. 1). Thus, in compression tests, the water holding capacity of agarose, together with the weak compressibility of water, may explain that the highest agarose content was associated to the highest elastic moduli. On the contrary, tensile tests reinforce the idea that these gels are anisotropic. When nanostructured hydrogels are subjected to tensile stress, the presence of agarose molecules attached to the fibrin fibers, as revealed by SEM, could reduce the ability of fibrin to distribute the load across the network, thus limiting the sequential 


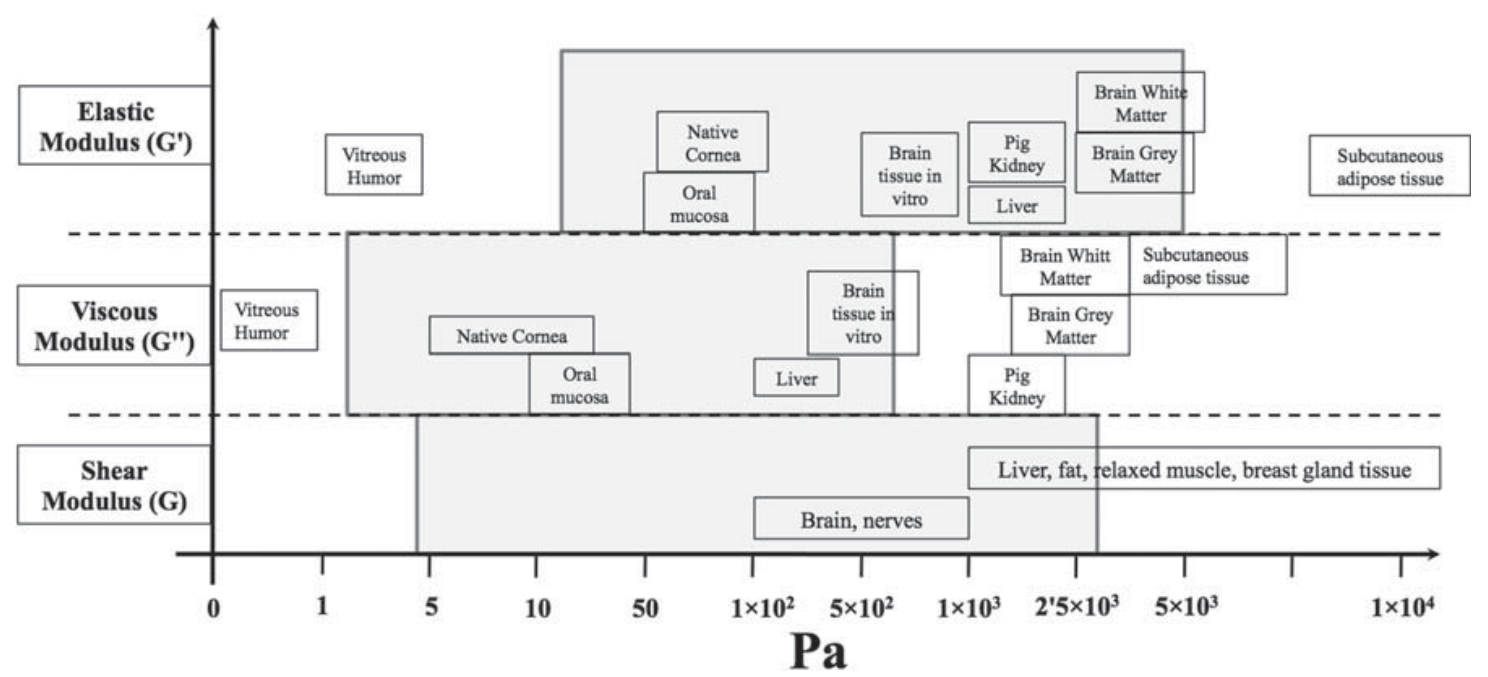

FIGURE 11. Values of elastic, viscous, and shear moduli of different native tissues. Shadowed rectangles represent the ranges covered by our FA hydrogels. Data for native tissues are taken from Refs. 19-24.

stiffening capability of fibrin hydrogels. On the other hand, the presence of non-homogeneities, derived from the addition of agarose, could influence the tensile tests results, which are very sensitive to any irregularities in the biomaterial, which is homogeneous otherwise. In the case of shearing tests, the samples are subjected to shearing forces in a plane perpendicular to the direction gravity and nanostructuration forces (Fig. 1). Very likely, the presence of waterswelled agarose reinforces the connection between different layers of fibrin fibers, which otherwise will be more free to slip one over another.

\section{Potential applications of FA tissue-like hydrogels in} tissue engineering

Both fibrin and agarose are materials that can be used as cell culture substrates and/or scaffolds due to their biocom- patibility and cell binding sites. Thus, from the point of view of cell adhesion and biocompatibility, fibrin and FA hydrogels can be safely used as scaffolds for the regeneration of tissues. Nevertheless, potentiality for precise applications will depend on the biomechanical properties of the native tissue to be replaced, which should be matched by those of the artificial hydrogels. Previous works demonstrated that the values of the viscoelastic moduli of nanostructured and non-nanostructured FA hydrogels at $0.1 \%$ of agarose are comparable to the ones of porcine cornea and human vocal fold mucosa, respectively. ${ }^{9,14}$ Our present study demonstrates that it is possible to adjust the biomechanical properties of fibrin and FA hydrogels within a broad range, by changing the agarose content and the hydration gradethe Young's Modulus ranges between 5 and $460 \mathrm{kPa}$, the compression elastic modulus between 1 and $75 \mathrm{kPa}, G$

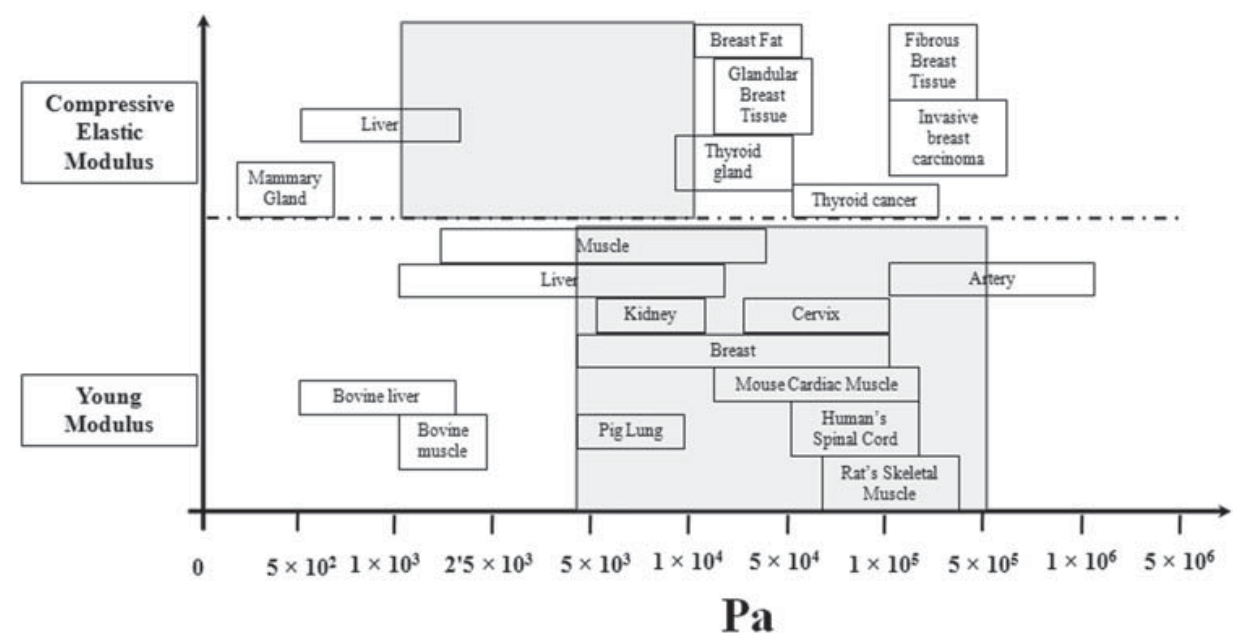

FIGURE 12. Values of compressive elastic modulus and Young's modulus of different native tissues. Shadowed rectangles represent the ranges covered by our FA hydrogels. Data for native tissues are taken from Refs. 26 and ${ }^{27}$ and references therein. 
between 4 and $3100 \mathrm{~Pa}, G^{\prime}$ between 15 and $4750 \mathrm{~Pa}$ and $G^{\prime \prime}$ between 2 and $600 \mathrm{~Pa}$. The adjustability of their biomechanical properties makes fibrin and FA hydrogels potential candidates for a good number of applications in tissue F12 F11 engineering (Figs. 11 and 12).

As observed in Figure 11, the viscoelastic moduli of some native tissues, as determined by previous reports, fall within the ranges covered by our fibrin and FA hydrogels. ${ }^{19-24}$ Human tissues from cornea, oral mucosa, nerves, and liver present viscoelastic moduli in the range of the fibrin and FA hydrogels analyzed in this work, suggesting that the FA technology could be used for the efficient replacement of these tissues form a mechanical standpoint. ${ }^{9,15,24}$ Another potential application of fibrin and FA hydrogels is their use as soft tissue fillers in surgery, an application which would benefit from the high water holding capacity of agarose. ${ }^{10}$ Note that the viscoelastic moduli of our fibrin and FA hydrogels cover those values reported for commercial soft tissue fillers. ${ }^{25}$ According to the values of their tensile Young's modulus and compressive elastic modulus, our fibrin and FA hydrogels also match the properties of a number of native tissues, including liver, muscle, kidney, cervix, breast and artery, supporting again the potential usefulness of these biomaterials (Fig. 12)-see Refs. 26 and ${ }^{27}$ and references therein.

In addition to these human tissues, fibrin and FA hydrogels might be successfully used as scaffolds for the regeneration of other human tissues. For example, although the values of the elastic shear modulus of our fibrin and FA hydrogels are lower than those reported in literature for native cartilage $\left(G^{\prime} \sim 0.03 \mathrm{MPa}\right),{ }^{28}$ the use of agarose content above the $0.5 \%$ used as a maximum in our work, could possibly contribute to the generation of hydrogels appropriate for cartilage regeneration. In fact, there are studies where fibrin and agarose scaffolds are used for cartilage regeneration. ${ }^{29}$ Fibrin and FA hydrogels could also be used for the regeneration of damaged skin or as subcutaneous adipose tissue, even though the value of the viscoelastic moduli of skin tissues $\left(G^{\prime} \sim 2 \mathrm{MPa}\right)$ or subcutaneous adipose tissue $\left(G^{\prime} \sim 104 \mathrm{~Pa}\right.$; $\left.G^{\prime \prime} \sim 103 \mathrm{~Pa}\right)$ are higher than the ones measured for FA. ${ }^{20}$ Again, the viscoelastic moduli of FA hydrogels could probably be increased above the values reported in this work by increasing the agarose content. Future works should determine the usefulness and biocompatibility of these types of hydrogels for other applications.

\section{CONCLUSIONS}

In this work, we have analyzed the effects of water and agarose contents on the biomechanical properties of fibrin and FA tissue-like hydrogels. For this aim, we have performed tensile tests, compressive tests and shear tests. Results of our study demonstrated that the biomechanical properties of these hydrogels can be modulated within a broad range by adjusting the agarose and water contents. The combination of nanostructuration and agarose contents allowed adjusting the biomechanical properties of FA hydrogels within a broad range, matching the properties of a good number of native tissues. The adjustability of the mechanical properties of FA hydrogels, together with their inherent biocompatibility, opens the possibility of a broad range of applications in medicine, where biocompatible, biodegradable, and natural biopolymers with high resistance are required. Cell viability studies with different cells sources should be performed in the near future in order to determine the potential usefulness of FA tissue-like hydrogels for these applications.

\section{REFERENCES}

1. Drury JL, Mooney DJ. Hydrogels for tissue engineering: Scaffold design variables and applications. Biomaterials 2003;24:43374351.

2. Orwin EJ, Borene ML, Hubel A. Biomechanical and optical characteristics of a corneal stromal equivalent. J Biomech Eng 2003;125: 439-444.

3. Ahearne M, Yang Y, Liu K-K. Mechanical characterisation of hydrogels for tissue engineering applications. Topics Tissue Eng 2008.

4. Lee KY, Mooney DJ. Hydrogels for tissue engineering. Chem Rev 2001;101:1869-1879.

5. Mosesson MW, Siebenlist KR, Meh DA. The structure and biological features of fibrinogen and fibrin. Ann N Y Acad Sci 2001;936: $11-30$.

6. Mosesson MW. Fibrinogen and fibrin structure and functions. Thromb Haemost 2005;3:1894-1904.

7. Ahmed TA, Dare EV, Hincke M. Fibrin: A versatile scaffold for tissue engineering applications. Tissue Eng Part B Rev 2008;14:199-215.

8. Alaminos M, Del Carmen Sanchez-Quevedo M, Munoz-Avila JI, Serrano D, Medialdea S, Carreras I, et al. Construction of a complete rabbit cornea substitute using a fibrin-agarose scaffold. Invest Ophthalmol Vis Sci 2006;47:3311-3317.

9. Ionescu AM, Alaminos M, De la Cruz Cardona J, De Dios GarciaLopez Duran J, Gonzalez-Andrades M, Ghinea R, et al. Investigating a novel nanostructured fibrin-agarose biomaterial for human cornea tissue engineering: Rheological properties. J Mech Behav Biomed Mater 2011;4:1963-1973.

10. Stellwagen J, Stellwagen NC. Internal structure of the agarose gel matrix. J Phys Chem 1995;99:4247-4251.

11. Sanchez-Quevedo MC, Alaminos M, Capitan LM, Moreu G, Garzon I, Crespo PV, et al. Histological and histochemical evaluation of human oral mucosa constructs developed by tissue engineering. Histol Histopathol 2007;22:631-640.

12. Carriel V, Garzon I, Jimenez JM, Oliveira AC, Arias-Santiago S Campos A, et al. Epithelial and stromal developmental patterns in a novel substitute of the human skin generated with fibrinagarose biomaterials. Cells Tissues Organs 2012;196:1-12.

13. Carriel V, Garrido-Gómez J, Hernández-Cortés P, Garzón I, GarcíaGarcía S, Sáez-Moreno JA, et al. Combination of fibrin-agarose hydrogels and adipose-derived mesenchymal stem cells for peripheral nerve regeneration. J Neural Eng 2013;10:026022.

14. Rodriguez IA, Lopez-Lopez MT, Oliveira AC, Sanchez-Quevedo MC, Campos A, Alaminos M, et al. Rheological characterization of human fibrin and fibrin-agarose oral mucosa substitutes generated by tissue engineering. J Tissue Eng Regen Med 2012;6:636644.

15. Chan RW, Titze IR. Viscoelastic shear properties of human vocal fold mucosa: Measurement methodology and empirical results. $J$ Acoust Soc Am 1999;106:2008-2021.

16. Guthold M, Liu W, Sparks EA, Jawerth LM, Peng L, Falvo M, et al. A comparison of the mechanical and structural properties of fibrin fibers with other protein fibers. Cell Biochem Biophys 2007; 49:165-181.

17. Piechocka IK, Bacabac RG, Potters M, Mackintosh FC, Koenderink $\mathrm{GH}$. Structural hierarchy governs fibrin gel mechanics. Biophys $J$ 2010;98:2281-2289.

18. Hudson NE, Houser JR, O'Brien ET III, Taylor RM II, Superfine R, Lord ST, et al. Stiffening of individual fibrin fibers equitably distributes strain and strengthens networks. Biophys J 2010;98:16321640 . 
19. Sharif-Kashani P, Hubschman J-P, Sassoon D, Kavehpour HP Rheology of the vitreous gel: Effects of macromolecule organization on the viscoelastic properties. J Biomech 2011;44:419-423.

20. Geerligs M, Peters G. Linear viscoelastic behavior of subcutaneous adipose tissue. Biorheology 2008.

21. Green MAM, Bilston LE, Sinkus R. In vivo brain viscoelastic properties measured by magnetic resonance elastography. NMR Biomed 2008:755-764.

22. Nasseri S, Bilston L, Phan-Thien N. Viscoelastic properties of pig kidney in shear, experimental results and modelling. Rheol Acta 2002:180-192.

23. Klatt D, Hamhaber U, Asbach $P$, Braun J, Sack I. Noninvasive assessment of the rheological behavior of human organs using multifrequency MR elastography: A study of brain and liver viscoelasticity. Phys Med Biol 2007;52:7281-7294.

24. Vanderhooft JL, Alcoutlabi M, Magda JJ, Prestwich GD. Rheological properties of cross-linked hyaluronan-gelatin hydrogels for tissue engineering. Macromol Biosci 2009;9:20-28.
25. Sundaram H, Voigts B, Beer K, Meland M. Comparison of the rheological properties of viscosity and elasticity in two categories of soft tissue fillers: Calcium hydroxylapatite and hyaluronic acid. Dermatol Surg 2010;36(Suppl 3):1859-1865.

26. Wells PNT, Liang H-D. Medical ultrasound: Imaging of soft tissue strain and elasticity. J R Soc Interface 2011:8:15211549.

27. Levental I, Georges PC, Janmey PA. Soft biological materials and their impact on cell function. Soft Matter 2007;3:299-306.

28. Stolz M, Raiteri R, Daniels AU, Van Landingham MR, Baschong W, Aebi U. Dynamic elastic modulus of porcine articular cartilage determined at two different levels of tissue organization by indentation-type atomic force microscopy. Biophys J 2004;86: 3269-3283.

29. Chien $\mathrm{C}$, Ho H, Liang $\mathrm{Y}-\mathrm{C}$, Ko $\mathrm{P}$, Sheu M-T, Chen $\mathrm{C}-\mathrm{H}$. Incorporation of exudates of human platelet-rich fibrin gel in biodegradable fibrin scaffolds for tissue engineering of cartilage. J Biomed Mater Res B Appl Biomater 2012;100:948-955. 